\title{
Development of flaxseed gum/konjac glucomannan with agar as gelling agents with enhanced elastic properties
}

\author{
Ji Soo Yang ${ }^{1+}$, Junghoon Kim ${ }^{4,5+}$, Jungwoo Hahn ${ }^{2 *}$, \& Young Jin Choi ${ }^{1,2,3 *}$ \\ 1 Department of Agricultural Biotechnology, Seoul National University, 1 Gwanakro, Gwanakgu, Seoul \\ 08826, Korea; annayang@snu.ac.kr (J. S. Y.); e-mail@e-mail.com \\ 2 Center for Food and Bioconvergence, Seoul National University, 1 Gwanakro, Gwanakgu, Seoul 08826, \\ Korea \\ 3 Research Institute of Agriculture and Life Sciences, Seoul National University, 1 Gwanakro, Gwanakgu, \\ Seoul 08826, Korea \\ 4 Department of Food Science and Biotechnology, Sejong University, 209 Neungdong-ro, Kwangjin-gu, Seoul, \\ 05006, Korea; david@intakefoods.kr (J. K.); e-mail.com \\ 5 Intake, 116 Myeongdal-ro, Seocho-gu, Seoul, 06659, Korea \\ * Correspondence: wjddnek1@snu.ac.kr, choiyj@snu.ac.kr; Tel.: (+82 2880 4858, +82 2880 4851) \\ + These authors contributed equally to the work.
}

\begin{abstract}
In this study, a natural-based gelling agent comprised of blended flax seed gum (FSG), konjac glucomannan (KG), and agar gel (AG) was developed for application to control the textural properties of foods. The compound gels, including FSG, KG, and AG, were investigated to determine their physicochemical properties, including minimum gelling concentration, water binding capacity, water soluble index, and swelling power. In addition, we analyzed the rheological properties of the compound gel through texture analysis, frequency sweep, and creep and recovery. The microstructure of the compound gel was identified and compared with the viscoelastic properties of the gel. Overall, these results showed that the F4K6 (4:6:2 of FSG:KG:AG) could serve as an excellent gelling agent, which endowed food gel with the promoted elastic properties, water capacity, and rigid surface morphology. This work suggests that novel gelling agents, including FSG, KGM, and AG, successfully prepared food gels with improved physicochemical properties.
\end{abstract}

Keywords: flaxseed gum; konjac glucomannan; agar; gelling agent

\section{Introduction}

Hydrocolloids, which are primarily composed of many hydroxyl groups, are highmolecular-weight compounds, and they usually are used as thickeners, texturizers, stabilizers, and gelling agents [1]. Gelling agents can provide some juicy foods with elastic properties through the formation of a gel. Comparatively few gums can form gels, and there is an even more limited number of gums applied to foods. The gelling agents that are used frequently in foods can be classified broadly into polysaccharides and proteins, including natural gums, pectin, agarose, gelatin, whey proteins, zein, and starches. The main reason for using hydrocolloids as a gelling agent in food is their ability to control the rheology of the food system, which can be represented by two basic properties of the food system, i.e., viscous properties and solid mechanical properties. Also, such gelling agents are used to transform the sensory properties by controlling the rheological food system. However, efficiently providing solid properties to water-rich foods entails a significant challenge in terms of the gelling agent's structural aspects. It must have high water binding and water holding capacities and requires strong syneresis [2]. Accordingly, several studies have been performed using combinations of various hydrocolloids, taking into account the role of controlling viscosity and elasticity in the foods [3]. 
Flaxseed (Linum usitatissimum L.), which contains a substantial quantity of water soluble polysaccharide (mucilage) in the outermost layer of flaxseed hulls, is used extensively for oil, fiber, and food. Flaxseed gum (FSG) is extracted easily from flaxseed by various solvents, including water and ethanol. Flaxseed gum consists of a mixture of $25 \%$ acidic rhamnogalacturonans-containing low-molecular-pectin and 75\% neutral arabinoxylans [4,5]. FSG is a neutral heteropolysaccharide that is composed of xylose, galctose, rhamnose, arabinose, fucose, glucose, and galacturonic acid [6,7]. In the food industry, it is considered to be a good hydrocolloid thickener [8] and gelling agent [9]. FSG has many advantages, such as an easy separation process, good water-holding capacity, good waterbinding capacity [10], and low viscosity [11], so if its poor elasticity can be overcome, it possibly could be used as a novel natural gelling agent.

Konjac gluconmannan (KGM), which is a natural, water-soluble polysaccharide, can be extracted from the tubers of Amorphophallus konjac plants [12]. KGM is a copolymer of irregular interaction of $\beta$-1, 4-linked d-mannose, and d-glucose with the molar ratio of 1.6:1 [13,14]. KGM is used extensively as an emulsifier, a thickener, a stabilizer, and as a gelling agent [15]. KGM can form high-concentration gels in strong alkaline conditions, but there are some limitations associated with these gels, including the large volumes of KGM required and their poor taste. So, these limitations must be improved in order to overcome some of their inherent shortcomings and to broaden their application possibilities. However, KGM requires a high concentration and strong alkaline conditions to form a gel. Therefore, it must be improved to overcome its inherent shortcomings and broaden its versatility.

Agar is a colloid that can be extracted from red algae (Gelidiaceae and Gracilariaceae), and it currently is used as a gelling, thickening, and stabilizing additive [16]. Agar consists of a mixture of two components, i.e., 1) 70\% agarose of the linear polysaccharide as agarobiose, D-galactose, and 3,6-anhydro-L-galactopyranose and 2) agaropectin, a heterogeneous mixture of smaller molecules, such as D-galactose and L-galactose, modified with acidic side-groups [17].

Based on the properties described above, we proposed that FSG/KGM blends with AG could develop as novel gelling agents to enhance the elastic properties of water-rich foods. The addition of KGM and a small amount of AG could improve the gel properties of the FSG system and overcome the flaws in the physicochemical properties of individual gelling agents, such as FSG, KGM, and AG. In this study, the synergism among FSG, KGM, and AG was investigated by TPA, Rheological measurement, SEM, and FT-IR. The gelling agents that were developed were adjusted to these three samples' ratios, and they were analyzed structurally via SEM.

\section{Materials and Methods}

\subsection{Chemicals, reagents, and instruments}

Flaxseed was purchased from Handsherb Co. (Yeongcheon, Korea), and Konjac glucomannan powder was purchased from Haenafood Co. (Seoul, Korea). Agar powder was obtained from Woori-ga Co. (Yangju, Korea).

Texture profile analyses (TPAs) of the proposed gels were obtained using a Texture Analyzer TA-XTplus/30 (Stable Micro Systems, UK). The rheology behavior of the gel samples was analyzed using a HAAKE RheoStress 1 Rheometer (Thermo Fisher Scientific, USA). The micromorphologies of the hydrogels were analyzed using the scanning electron microscope (SEM, JEOL-5410 LV) at Seoul National University's National Instrumentation Center for Environmental Management (NICEM). 


\subsection{Extraction of flaxseed gum}

We optimized the separation process to obtain a gum mucilage from natural whole flaxseed according to [18] with some modifications. Since choosing the method to be used for the mechanical extraction of the gum solution from flaxseed is very important due to technical issues, such as the extraction of other substances, we finally decided to use the whole seeds in a hot water extraction process [5]. First, the flax seeds were placed in hot, deionized water $\left(90^{\circ} \mathrm{C}\right)$ at a flaxseed-to-water concentration of $7.7 \%(\mathrm{w} / \mathrm{w})$, and they were boiled for $10 \mathrm{~min}$ with continuous gentle stirring. The gum solution was filtered from the heat-treated mixture by a sieve that had a 14-mesh Tyler Screen $(1.3 \mathrm{~mm})$ (NAVIMRO, Korea) and precipitated in three volumes of $95 \%$ ethanol. The precipitated gum ( $\mathrm{pH} 7.0$ ) was collected by centrifugation, dispersed in distilled water, and dried overnight at $65{ }^{\circ} \mathrm{C}$ in a dry oven. Then, the dried gum was ground into fine particles $(0.1 \mathrm{~mm})$ by a mixer before it was used in the experiment. The yield of FSG was determined using the following equation:

$$
\text { Yield }(\%)=(\text { Dried gum }) /(\text { Flaxseed }) \times 100
$$

where "Flaxseed" represented the total mass of the water-soluble portion of the flaxseed in g after drying (dry weight), and "Flaxseed gum" represented the mass of flaxseed in $g$ (dry weight). The separation yield of FSG was about $15 \%$.

\subsection{Preparation of the gel samples}

The polysaccharide solutions were prepared at a constant concentration of $2.4 \mathrm{~g} / 100$ $\mathrm{mL}(2.4 \% \mathrm{w} / \mathrm{v})$, which contained FSG/KGM $(2.0 \% \mathrm{w} / \mathrm{v})$ with different ratios and a fixed concentration of AG $(0.4 \% \mathrm{w} / \mathrm{v})$. The compound gels with FSG/KGM at different ratios, i.e., 10:0, 8:2. 6:4, 4:6, 2:8, and 0:10 with AG added at a constant ratio of 2, were denoted as FK10, FK8, FK6, FK4, FK2, and FK0, respectively. In addition, neat FSG gel (F12) and neat KGM gel (K12) were prepared as a control samples.

The compound gels were prepared at a constant concentration of $1.2 \mathrm{~g} / 50.0 \mathrm{~mL}$. First, FSG powder, KGM powder, and AG powder were added into a 50-mL glass vial according to the ratio, and then mixed for about 1 minute using a stirrer bar. Then, deionized water was added to the vial and continuously homogenized, followed by stirring at $95^{\circ} \mathrm{C}$ for $30 \mathrm{~min}$ to prepare the solutions. The dispersed solutions were transferred to a silicone mold $(2.2 \mathrm{~cm}$ wide $\times 2.2 \mathrm{~cm}$ long $\times 2.5 \mathrm{~cm}$ high) and molded with a volume of about 12 $\mathrm{mL}$. To prepare the samples, they were left sequentially at room temperature for 30 minutes, refrigeration temperature $\left(4^{\circ} \mathrm{C}\right)$ overnight, and again at room temperature for more than $2 \mathrm{~h}$.

In addition, in the experiment to determine the minimum AG concentration, we measured the height/width ratios of the cuboid gel samples that were prepared using the proposed gel preparation method.

\subsection{Minimum gelling concentration}

A series of FSG/KGM/AG mixtures was prepared by varying the concentration of the gum powder $(0.6,0.8,1.0,1.2$, and $1.4 \% \mathrm{w} / \mathrm{v})$ to observe the gelling behavior of the compound gels. All of the mixtures were heated at $95{ }^{\circ} \mathrm{C}$ for $20 \mathrm{~min}$. The samples were cooled 
at room temperature and then refrigerated overnight at $4{ }^{\circ} \mathrm{C}$. Afterwards, they were kept at room temperature for $2 \mathrm{~h}$. The gelling behavior was determined by observing an inverted tube to determine the range of the concentrations in which the gel did not flow under the force of gravity.

\subsection{Physicochemical properties of the compound gels}

To confirm the physicochemical properties of the compound gels, their water binding capacities (WBCs), water solubility indices (WSIs), and swelling powers (SPs) were measured, and, finally, the minimum gelling concentrations were optimized. The gels were made according to the procedure suggested above. The WSI and SP values of the compound gels were obtained using the procedure described by Jiang et al. (2019) with some modification. Each sample $(0.115 \mathrm{~g})$ was mixed in $30 \mathrm{~mL}$ of distilled water in $50-\mathrm{mL}$ centrifuge tubes $\left(90^{\circ} \mathrm{C}, 30 \mathrm{~min}\right)$, followed by centrifugation at 20,000 $\mathrm{g}$ for $30 \mathrm{~min}$.

\subsubsection{Water binding capacity}

WBC was defined as the \% concentration of the weight of the sediment relative to the weight of the sample, and it was calculated according to Eq. (2):

$$
\text { WBC }(\%)=((\text { W4-W3 }) / \text { weight of sample }) \times 100
$$

where W3 and W4 in Eqs. (2) and (4) are the weight of the tube and the weight of the tube with the sediment, respectively, and 'W4-W3' represents the weight of the sediment.

\subsubsection{Water solubility index and swelling power}

The WSI and SP of the blended gel samples were determined by the procedure of Jiang et al. (2019) with slight modification. The WSI and SP were calculated by Eqs. (3) and (4), respectively:

$$
\begin{aligned}
& \text { WSI }(\%)=((\mathrm{W} 2-\mathrm{W} 1) / \text { weight of dried sample }) \times 100 \\
& \text { SP }(\mathrm{g} / \mathrm{g})=(\mathrm{W} 4-\mathrm{W} 3) /(\text { weight of dried sample } \times(100-\mathrm{WSI}))
\end{aligned}
$$

where W1 and W2 are the weight of the empty petri dish and the weight of the petri dish with the dried residue, respectively, and 'W2-W1' represents the weight of the dried supernatant.

\subsection{Texture profile analysis}

The samples used for Texture profile analysis (TPA) were prepared by the procedure used to prepare gel samples. Texture profile analysis was done at room temperature (apporoximately20 ${ }^{\circ} \mathrm{C}$ ). The samples were penetrated with a TA11/1000 probe. Each sample was compressed twice at a deformation of $40 \%$ of the height of the sample and at a speed of $1 \mathrm{~mm} / \mathrm{s}$ with the control force of $5 \mathrm{~g}$-force, and the samples' textural parameters were recorded, included hardness, cohesiveness, springiness, chewiness, and gumminess.

\subsection{Rheological study}

To analyze the rheological properties of the gels, we performed the frequency sweep test and the creep and recovery test. The temperature of the rheometer (HAAKE RheoStress 1) was controlled at $20^{\circ} \mathrm{C}$ by a water bath connected to the Peltier system in the bottom plate. A strain sweep experiment was performed to determine the linear viscoelastic regions of each sample, and, finally, the region was determined at $1 \mathrm{~Hz}(\mathrm{rad} / \mathrm{s})$. The viscoelastic properties of the compound gel mixtures were measured within the linear 
viscoelastic region. The sample was placed on the bottom plate, and the parallel plate was lowered immediately to set size of the gap at $5 \mathrm{~mm}$. A moderate amount of a petroleum jelly was used to prevent evaporation. An equilibration period of $2 \mathrm{~min}$ was maintained before each measurement.

\subsubsection{Frequency sweep tests}

The oscillatory frequency sweep tests were performed at $10{ }^{\circ} \mathrm{C}$ over the angular frequency range from 0.01 to $1 \mathrm{~Hz}$. The amplitude of the shear stress was fixed at $1 \mathrm{~Pa}$. The amplitude of the strain was chosen as $5 \%$ based on the strain sweep results so that it would be in the linear viscoelastic region for all of the samples. A parallel plate (a cone and a plate) shape was selected, and it was made of stainless steel (diameter $40 \mathrm{~mm}$, spacing $1 \mathrm{~mm}$ or $1 \mathrm{~mm}$ gap for a $40-\mathrm{mm}$ cone and plate geometry). The storage modulus $\left(G^{\prime}\right)$, loss modulus $\left(G^{\prime \prime}\right)$, $\tan \delta$, and $\tan \delta$ were determined as functions of frequency.

\subsubsection{Creep and recovery test}

It is necessary to optimize the test conditions (temperature, stress level, loading time) for creep and recovery experiments of gels with different stiffness for example, the conditions should be optimized when the gel would reach the zero level of steady state and/or a practical waiting time could be required. Therefore, based on the optimized conditions, the change in shear strain versus an applied instantaneous stress (30 Pa) was measured for $300 \mathrm{~s}$, after which the stress was removed and the change in strain was recorded for an additional $300 \mathrm{~s}$. An aluminum parallel plate geometry (diameter $40 \mathrm{~mm}$, spacing $1 \mathrm{~mm}$ ) was selected for creep recovery measurements, and all creep and recovery experiments were conducted at $20^{\circ} \mathrm{C}$. The creep and recovery results were described in terms of the elasticity $(\%)$ and the shear compliance function, $J(t)$.

$$
\begin{aligned}
& \text { Elasticity }(\%)=\left(\frac{J_{\max }-J_{\min }}{J_{\max }}\right) \times 100 \\
& J(t)=\frac{1}{G_{1}}+\frac{1}{G_{2}}\left[1-\exp \left(-\frac{G_{2}}{\eta_{2}} t\right)\right]+\frac{t}{\eta_{1}}
\end{aligned}
$$

where $J_{\max }$ is the maximum compliance; $J_{\min }$ is the permanent deformation; $G_{1}$ is the elastic modulus of the Maxwell unit; $G_{2}$ is the instantaneous elastic modulus of Kelvin-Voigt; $\eta_{1}$ is the residual viscosity of the Maxwell unit; and $\eta_{2}$ is the internal viscosity.

\subsection{Scanning electron microscopy observation}

Before the observation, the compound gels were lyophilized by a vacuum freeze dryer at $-40{ }^{\circ} \mathrm{C}$. The samples were fixed on a microscope stub and then coated with a platinum layer with a thickness of $15 \mathrm{~nm}$. The samples were observed by SEM with a potential accelerator of $2 \mathrm{kV}$ and magnified by $300 \mathrm{x}$ and $2000 \mathrm{x}$.

\subsection{Fourier transform infrared spectroscopy (FIIR)}

The infrared spectral patterns of the compound gels were investigated using an FTIR spectrometer (Frontier, PerkinElmer, Inc., USA) through the $\mathrm{KBr}$ compression pellet method [19]. Each sample was fragmented and mixed uniformly with $\mathrm{KBr}$. Also, the samples were shaped into pellets prior to analysis. The calibration of the instrument was performed using $\mathrm{KBr}$ pellets as the reference, and the infrared spectra were documented within the range of 4000 to $500 \mathrm{~cm}^{-1}$.

\subsection{Statistical analyses}


Statistical analysis of the results was performed using a Statistical Package for the Social Sciences System (SPSS Statistics 25). Duncan's multiple range test was used to identify significant differences $(P<0.05)$ between means. The results were reported as mean \pm standard deviation.

\section{Results and discussion}

\subsection{Optimization of the concentrations}

In order to confirm the possibility of hydrogel containing FSG, KGM, and AG as a novel gelling agent capable of showing high elasticity, we had to set the concentration of each component and the total concentration range of the polysaccharides used. Therefore, the concentration of AG, which represented a more solid property than the other two components, and the minimum gelling concentration were confirmed (Figure 1).

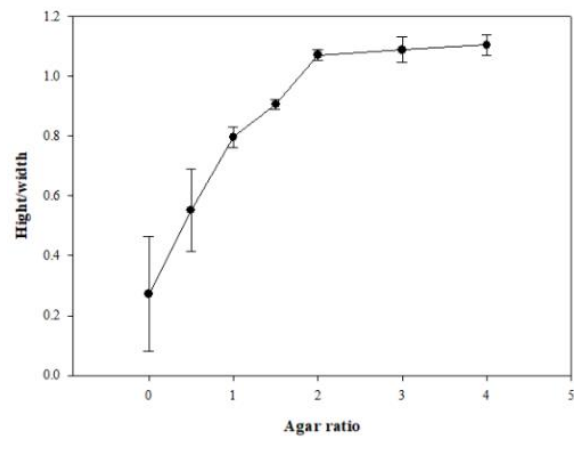

(A)

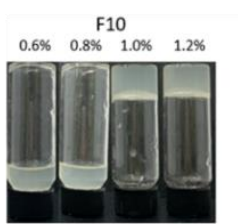

F4K6

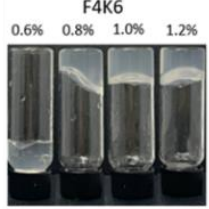

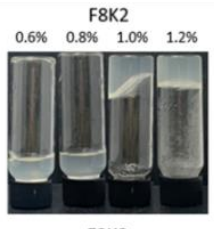

F2K8

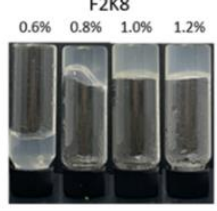

(B)

Figure 1. Optimization of concentrations for showing the solid property of compound gels: (A) Curves of length/width of FSG/KGM compound gels $(2.4 \% \mathrm{w} / \mathrm{v})$ with different agar ratios; (B) Appearance of the inverted compound gels with different concentrations for determining minimum gelling concentrations.

\subsubsection{Optimization of the concentration of the agar gel}

We determined the minimum percentage of AG contained in the proposed gel based on the result of a preliminary experiment that indicated that AG was essential for the formation of a rigid structure. As shown in Figure 1A, as the ratio of AG increased, the height/width value increased. However, the height/width value remained constant when the ratio of AG was 2 or more. As the ratio of AG increased, a gel was formed that had a rigid structure, but the brittleness of the gel also increased. Based on these results, we fixed the ratio of agar at $2(0.4 \% \mathrm{w} / \mathrm{v})$ to prepare gels that contained different ratios of FSG and KGM to investigate their solid properties.

\subsubsection{Minimum gelling concentration (MGC)}

Gelation occurs when the gums form a three-dimensional network that is resistant to flow under pressure. This ability to form gels is an important functional property in processing food and in the formulation of food for various products, such as jellies and many desserts. Gelling capacity frequently is measured by the minimum gelling concentration (MGC), which is defined as the lowest concentration required for a self-supporting gel to form. A lower MGC indicates that the sample has a better capacity to form gels [20]. 
Figure $1 \mathrm{~B}$ shows that the gels were formed at $1 \%(\mathrm{w} / \mathrm{v})$ in the cases of F10, F8K2, and F6K4, all of which contained relatively higher proportions of FSG. However, the samples in which the ratios of KGM were relatively higher, i.e., F4K6, F2K8, and K10, had lower MGCs, which indicated that the gels were formed at $0.8 \%(\mathrm{w} / \mathrm{v})$ against gravitational force. This indicated that the higher the ratio of KGM is in compound gels, the easier it is to retain the gel form by entrapping more water. Therefore, a smaller amount of gum powder could maintain the viscoelastic gel form in F4K6, F2K8, and K10 blend gels. Based on these results, the concentration of the total gum used was determined to be $2.4 \%(\mathrm{w} / \mathrm{v})$. Since this concentration is capable of forming a sufficiently rigid structure of the gel, it was used to investigate the physicochemical properties and microstructure analysis of the compound gel.

\subsection{Physicochemical properties of compound gels}

To confirm the physicochemical properties of compound gels, various measurement values were investigated, including WBC, WSI, and SP. These values of the compound gels that contained different levels of FSG and KGM were determined, and the results are presented in Table 1.

Table. Physicochemical properties of the compound gels with FSG, KGM, and AG

WBC was a water binding capacity. WSI was a water soluble index. SP was a solubility index. Values expressed were mean \pm standard deviation. Data of different alphabets in the same column were different with statistical significant $(p<0.05)$.

\subsubsection{Water binding capacity (WBC)}

WBC, which is one of the most basic values that can indicate the physicochemical properties of gel, is expressed as Eq. (2). It represents the percentage concentration of the

\begin{tabular}{cccc}
\hline Sample ID & \multicolumn{3}{c}{ Physicochemical parameter } \\
\cline { 2 - 4 } ( FSG/KGM/AG ratio) & WBC (\%) & WSI (\%) & SP (g/g) \\
\hline F10 (10:0:2) & $10.48 \pm 1.90^{\mathrm{a}}$ & $75.83 \pm 6.45^{\mathrm{b}}$ & $2.25 \pm 0.44^{\mathrm{a}}$ \\
F8K2 (8:2:2) & $17.36 \pm 1.65^{\mathrm{b}}$ & $64.17 \pm 10.33^{\mathrm{a}}$ & $2.56 \pm 0.60^{\mathrm{a}}$ \\
F6K4 (6:4:2) & $23.33 \pm 2.81^{\mathrm{c}}$ & $58.75 \pm 3.76^{\mathrm{a}}$ & $2.88 \pm 0.47^{\mathrm{a}}$ \\
F4K6 (4:6:2) & $24.96 \pm 4.22^{\mathrm{c}}$ & $59.17 \pm 6.65^{\mathrm{a}}$ & $3.12 \pm 0.63^{\mathrm{a}}$ \\
F2K8 (2:8:2) & $10.06 \pm 1.96^{\mathrm{a}}$ & $65 \pm 5.70^{\mathrm{ab}}$ & $1.450 .24^{\mathrm{a}}$ \\
K10 (0:10:2) & $9.80 \pm 2.39^{\mathrm{a}}$ & $95.83 \pm 3.03^{\mathrm{c}}$ & $1.44 \pm 4.73^{\mathrm{b}}$ \\
\hline
\end{tabular}

weight of the precipitate after external pressure treatment relative to the weight of the sample. As shown in Table 1, FK6 and FK4 showed high WBC values, i.e., 23.33\% and $24.96 \%$, respectively, which were significantly different $(p<0.05)$ from the values of the other samples. By contrast, the F10 and F2K8 samples showed the low WBC values of $10.48 \%$ and $10.06 \%$, respectively. WBC is related to the integrated hydrophilic ability of the composite gel that results from the structure, size, or composition of the gel [21,22], and it was confirmed that high hydrophilicity was exhibited when the KGM/WBC ratio was similar under this condition.

3.2.2. Water solubility index and swelling power 
According to Eq. (3), WSI presents the percent concentration of the dry weight of the supernatant to the total dry weight of the test sample. In other words, WSI indicates how soluble the gel sample is in water. As the KGM ratio increased, the WSI value decreased gradually. The WSI value of F10 was significantly higher $(p<0.05)$, and it could be confirmed that the solubility of the compound gel that included FSG, KGM, and AG was relatively low. KGM, which is composed of d-mannose and d-glucose, is hydrophilic and can exhibit strong polarity due to its large number of hydroxyl groups [23]. Therefore, KF0 could form more hydrogen bonds with water molecules [24], and the interacted structures of FSG, KGM, and AG were thought to show relatively low water solubility. In several studies, KGM has been found to exhibit synergistic hydrocolloid effects, such as improving gelling capacity with many substances, which was thought to be related to the low WSI of the compound gel [13].

The SP, including the meaning of WSI, can be represented by Eq. (4). In Eq. (4), SP represents the reciprocal of the percent concentration of the dry weight of the sediment relative to the weight of the sediment. In other words, SP means how much water the gel can hold or retain without being removed by an external force. This property has been reported to be related to the density and structure of the network as well as the polymersolvent interaction [25]. As KGM increased, the SP value tended to increase, and the F4K8 showed a significantly high SP value ( $\mathrm{p}<0.05)$. Taken together, the F6K4 and F4K6 samples that contained FSG and KGM in similar proportions showed good physicochemical properties as gelling agents.

\subsection{Texture properties of compound gels}

The texture that people perceive when they eat food has multi-dimensional properties. To quantify the properties of texture, the texture profile analysis (TPA), which is a simple and fast analysis technique that is used extensively in the food industry, can explore the possibilities of developing new foods or using different ingredients in foods. Table 2 shows the mechanical properties of compound gel systems based on the TPA of different ratios among FSG, KGM, and AG. In this study, hardness, cohesiveness, springiness, gumminess, and resilience were selected as the main textural parameters that depend on the properties of the gel, and analyses of the texture were performed on all test groups except for F12, which exhibited a sol behavior (i.e., weak-gel and/or liquid-like behavior). The hardness profile represented a tendency of increasing the hardness 1 and 2 , which generally represent the strengths applied to foods, including gels between the tongue and palate, as the proportion of KGM increases [26,27]. These results mean that higher strength was required for the deformation of the compound gel. However, F10 showed excessive decreases of hardness 1 and hardness 2, which would be judged as a result of the low density of the F10 structure due to the absence of KGM. In addition, as the proportion of KGM increased, cohesiveness tended to increase, which reflected the ability of the gels to recover their original state [28]. The values indicating the elasticity of the compound gels, including springiness, gumminess, and resilience, also tended to be higher as the ratio of KGM increased. All four parameters of TPA reflecting rheological properties, including cohesiveness, springiness, gumminess, and resilience, showed no significant difference in F4K6, F2K8, and $\mathrm{K} 10(p<0.05)$. It would be judged that, even though FSG had much lower elasticity than KGM, the compound gels of F4K6 and F2K8 could present as much elasticity as K10 through synergistic interaction among FSG, KGM, and AG. 


\begin{tabular}{ccccccc}
\hline $\begin{array}{c}\text { (FSG/KGM/AG } \\
\text { ratio) }\end{array}$ & Hardness 1 (g) & Hardness 2 (g) & Cohesiveness & $\begin{array}{c}\text { Springiness } \\
(\mathbf{m m})\end{array}$ & Gumminess (g) & Resilience \\
\hline F10 (10:0:2) & $352.75 \pm 58.33^{b c}$ & $263.00 \pm 76.44^{a b}$ & $0.25 \pm 0.02^{a}$ & $4.08 \pm 0.27^{a}$ & $86.50 \pm 14.84^{a}$ & $0.14 \pm 0.01^{a}$ \\
F8K2 (8:2:2) & $371.33 \pm 98.89^{b c}$ & $329.50 \pm 99.99^{b c}$ & $0.33 \pm 0.02^{a b}$ & $4.38 \pm 0.53^{a b}$ & $124.50 \pm 41.09^{a b c}$ & $0.18 \pm 0.02^{b}$ \\
F6K4 (6:4:2) & $359.67 \pm 57.77^{a b}$ & $324.67 \pm 47.51^{a b}$ & $0.38 \pm 0.06^{b}$ & $4.57 \pm 0.91^{a}$ & $141.00 \pm 39.84^{a b}$ & $0.19 \pm 0.02^{a b}$ \\
F4K6 (4:6:2) & $540.33 \pm 50.34^{c}$ & $494.33 \pm 49.66^{c}$ & $0.40 \pm 0.02^{b c}$ & $6.17 \pm 0.24^{b c}$ & $216.00 \pm 30.35^{b c}$ & $0.21 \pm 0.02^{b c}$ \\
F2K8 (2:8:2) & $556.67 \pm 41.28^{c}$ & $510.33 \pm 38.40^{c}$ & $0.43 \pm 0.02^{b c}$ & $6.48 \pm 0.81^{c}$ & $240.00 \pm 19.08^{c}$ & $0.23 \pm 0.01^{b c}$ \\
K10 (0:10:2) & $398.25 \pm 140.36^{b c}$ & $360.50 \pm 140.52^{b c}$ & $0.50 \pm 0.05^{c}$ & $6.10 \pm 0.86^{c}$ & $200.25 \pm 83.22^{b c}$ & $0.25 \pm 0.04^{c}$ \\
K12 (0:12:0) & $154.00 \pm 19.16^{a}$ & $154.33 \pm 18.77^{a}$ & $0.86 \pm 0.09^{d}$ & $3.83 \pm 0.82^{a}$ & $133.33 \pm 26.76^{a b}$ & $0.13 \pm 0.03^{a}$ \\
\hline
\end{tabular}

Table 2. Textural parameters of the compound gels including FSG, KGM, and AG

The values in Table 2 are mean \pm standard deviation. The differences between the data of different alphabets in the same column were statistically significant $(p<0.05)$.

\subsection{Rheological properties of compound gels}

The rheological characteristics of gels are of utmost importance, and they must be evaluated practically since the rheological characteristics are very closely correlated to the textural properties of products, which determine both sensory properties and acceptance by consumers. Therefore, we performed the rheological measurement of the gel through the frequency sweep test as small deformation testing and through the creep and recovery test as large deformation testing. Small deformation testing, which determines the shear or Young's moduli, is very useful for comparing the viscoelasticity of gelling systems. However, large deformation testing generally is used to measure the shear stress, yield stress, apparent viscosity, and the deformation and failure properties of gels. These two types of tests are not necessarily correlated, and they can provide complementary information.

\subsubsection{Frequency sweep tests}

Figure 2A shows that the $\mathrm{G}^{\prime}$ values, which are indicators of gel strength and elasticity, of all samples were higher than their $G^{\prime \prime}$ values, and both values showed a tendency to increase as the angular frequency increased. In addition, since the amount of KGM in compound gels increased, with the expectation of K12, the slopes of the $G^{\prime}$ graph showed a tendency to increase. In particular, the slope of the neat KGM hydrogel (K12) showed a steep increase; this was related to the exclusion of the AG that had a molecular helix, which could be aggregated, thereby resulting in large pores and rigid structures [29]. However, the compound gels that have high $G^{\prime}$ values would form a flexible and rigid network structure through hydrogen bonds among FSG, KGM, and AG, which strengthened the interaction between molecules [24]. However, from the perspective of FSG, as its proportion increased, the dynamic moduli decreased, and the decreasing slopes of the $\mathrm{G}^{\prime}$ curves in a high proportion of the FSG samples (F8K2 and F10) were slow. This result indicated that FSG would be associated with the formation of a flexible network, which was not destroyed by high strain [30,31]. This phenomenon may be attributed to the FSG and KGM molecular chains in compound gels that interact with the AG molecules by forming hydrogen bonds [32]. These interactions could reduce the aggregation of the AG helical structure and reduce the physical entanglement, resulting in more flexible networks. 


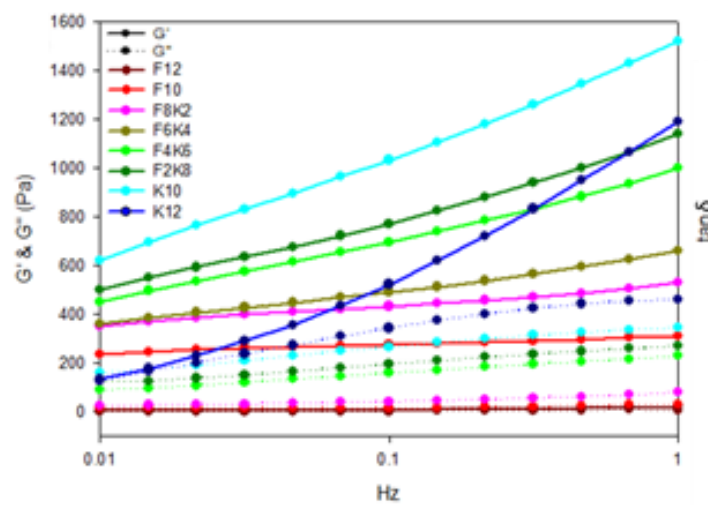

(A)

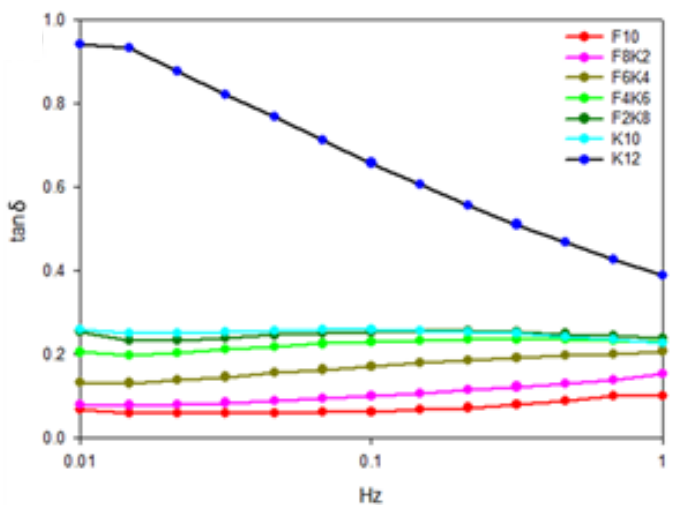

(B)

Figure 2. Frequency sweep curves of the blend gels: (A) Storage modulus $\left(\mathrm{G}^{\prime}\right)$ and loss modulus $\left(\mathrm{G}^{\prime \prime}\right)$; (B) tan $\delta$ of compound gels as a function of angular frequency $(0.01-1 \mathrm{~Hz})$.

The loss tangent, $\tan \delta$, which is equal to $G^{\prime \prime} / G^{\prime}$, intuitively reflected the dynamic viscoelastic behavior of the samples. In principle, when $\tan \delta<1$, samples mainly exhibit elastic properties [33]. Figure 2B shows that the values of tan $\delta$ of all hydrogels were less than 1, indicating that these gels had a stable network and were predominantly elastic. The $\tan \delta$ of K12 changed rapidly as the frequency increased due to the structure of the network being weaker than others. The increase of the FSG proportion decreased the value of $\tan \delta$, and the $\tan \delta$ values of F4K6, F2K8, and K10 were not significantly different. Consequently, $\tan \delta$ and the slopes of the curves both increased in the order of F4K6, F2K8, and K10, but they were not significantly different; two dynamic moduli of these gels were parallel, which meant that these gels showed a high gel strength, high elasticity, and solidlike gel behavior.

Table 3. Elasticity and the parameters of Burgers model of the compound gels

\begin{tabular}{cccc}
\hline $\begin{array}{c}\text { Sample } \\
\text { (FSG/KGM/AG } \\
\text { ratio) }\end{array}$ & Elasticity $(\%)$ & $\mathbf{G}_{\mathbf{1}}(\mathbf{P a})$ & $\mathbf{G}_{2}(\mathbf{P a})$ \\
\hline F12 $(12: 0: 0)$ & & & \\
F10 $(10: 0: 2)$ & $9.75^{\mathrm{a}}$ & $16.70^{\mathrm{a}}$ & $7.55^{\mathrm{a}}$ \\
F8K2 (8:2:2) & $3.99^{\mathrm{a}}$ & $271.14^{\mathrm{a}}$ & $2586.73^{\mathrm{c}}$ \\
F6K4 (6:4:2) & $48.89^{\mathrm{b}}$ & $468.88^{\mathrm{a}}$ & $1277.10^{\mathrm{c}}$ \\
F4K6 (4:6:2) & $58.38^{\mathrm{c}}$ & $523.40^{\mathrm{c}}$ & $1061.22^{\mathrm{b}}$ \\
F2K8 $(2: 8: 2)$ & $76.44^{\mathrm{d}}$ & $634.95^{\mathrm{cd}}$ & $1068.79^{\mathrm{b}}$ \\
K10 $(0: 10: 2)$ & $78.49^{\mathrm{d}}$ & $687.84^{\mathrm{cd}}$ & $972.13^{\mathrm{b}}$ \\
K12 $(0: 12: 0)$ & $77.44^{\mathrm{d}}$ & $783.67^{\mathrm{d}}$ & $1065.92^{\mathrm{b}}$ \\
\hline
\end{tabular}

The entries in the G1 column are the elastic moduli of the Maxwell units, and the entries in the G2 column are the instantaneous elastic moduli of Kelvin-Voigt. The values are 
expressed as mean \pm standard deviation. The data of different alphabets in the same column had different statistical significances $(p<0.05)$.

\subsubsection{Creep and recovery test}

We also confirmed the rheological property of the proposed gel through creep and recovery experiments. Unlike the frequency sweep test, the creep and recovery test could determine the viscoelastic properties of the gel by measuring the recovery (creep data) of the gel deformed by instantaneous stress as a creep compliance, J (strain/stress) [34]. The creep and recovery behaviors of the compound gels, including FSG, KGM, and AG, are shown in Figure 3. Samples, including F4K6, F2K8, and K10, showed similar maximum creep compliance $\left(J_{\max }\right)$. $J_{\max }$, which can explain how compliant the gels are, indicated that the higher the value, the easier it was to deform the gel [3]. However, the lower values of J represent stronger gel structures [35]. Figure 4 shows that F4K6, F2K8, and K10 had tendencies to deform less by instantaneous stress than other samples, and this result indicated that the structure of these gels was stronger and denser. When the load was removed, the strain under testing could be recovered partially to its original geometry, which can be expressed by recovery compliance $\left(J_{\mathrm{min}}\right.$, irreversible compliance), and the lower values of $J_{\mathrm{min}}$ indicate that the gel is more elastic. From the recovery curves, it seemed that F4K6, F2K8, and K10 would almost reach zero levels of residual strain $(J \min )$ asymptotically after a very long recovery time. In addition, we determined the Elasticity (\%) given by Eq. (5) using the compliances $\left(J_{\max }\right.$ and $\left.J_{\min }\right)$ (Table 3). The elasticities of F4K6, F2K8, and K10 exceeded 75\%, and they were significantly higher $(\mathrm{p}<0.05)$ than the elasticities of other gel samples. Thus, we were able to confirm that the gels with KGM ratios of 6 or more have rheological behaviors similar to an ideal elastic material.

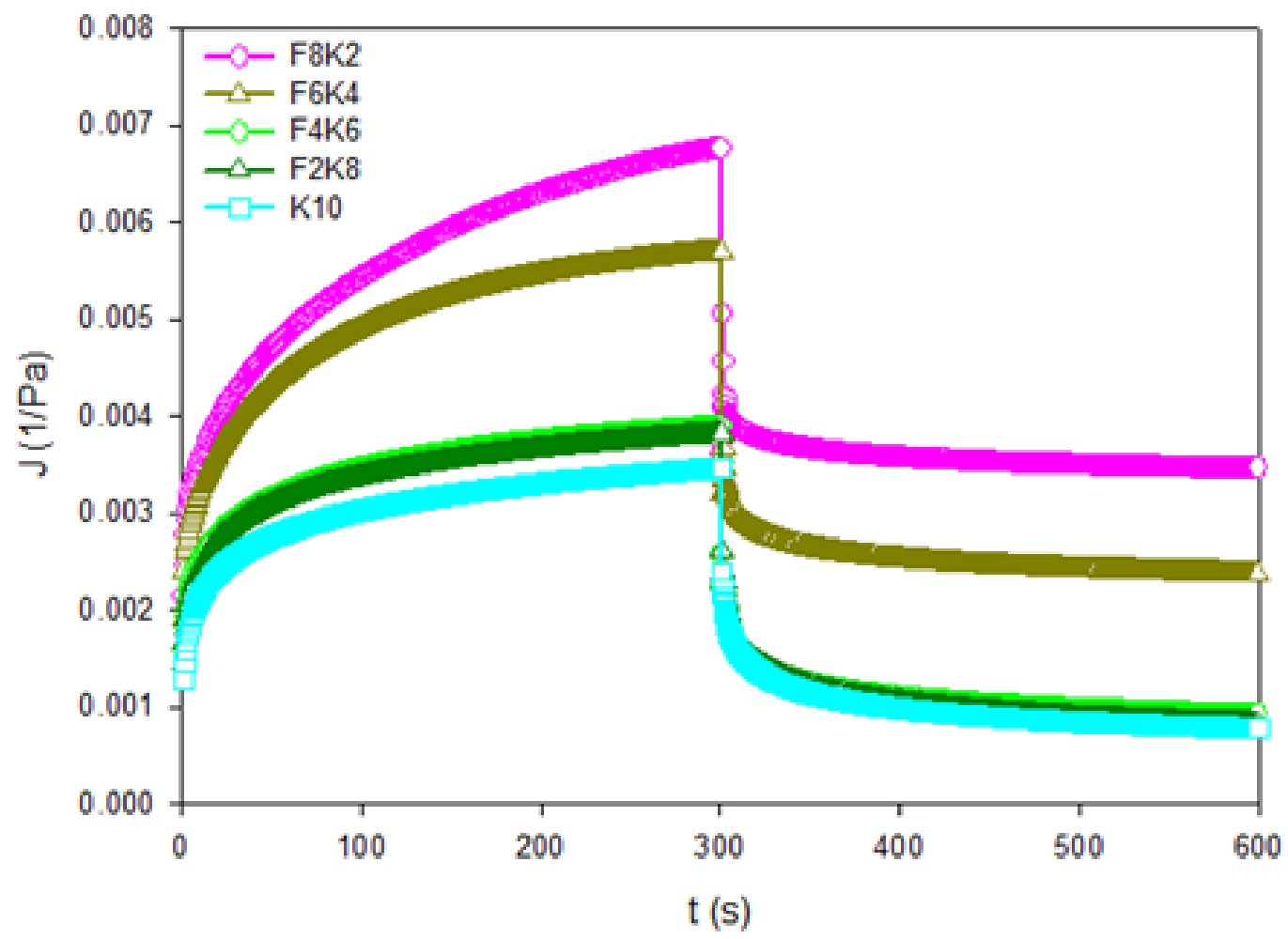

Figure 3. Representative creep and recovery compliance $(J)$ data of the compound gels: An instantaneous stress of $30 \mathrm{~Pa}$ was applied for $300 \mathrm{~s}$, and recovery was observed for an additional $300 \mathrm{~s}$. 
The Burgers model, given by Eq. 6, can explain creep and recovery behaviors mathematically. We expected to describe the creep and recovery behavior of the proposed gels satisfactorily through this model. Figure S1 shows that Burger's model consists of the Maxwell components and the Kelvin-Voigt components placed in series. The Maxwell element consists of a Newtonian dashpot placed in series with a Hookean spring with the elastic and viscous reactions occurring at different times. The Kelvin-Voigt element consists of a Newtonian dashpot placed parallel to a Hookean spring, and both the elastic and viscous reactions occur simultaneously. We applied Burgers model to the experimental data of the compound gels. The parameters of Burgers model were derived from Eq. (6), and they include $G_{1}$ and $G_{2}$, as shown in Table 3; $G_{1}$ is the instantaneous elastic modulus of the Maxwell spring, and $G_{2}$ is the retarded elastic modulus of Kelvin-Voigt (Barry, 1983). Table 3 shows that the value of $G_{1}$ increased gradually as the KGM ratio increased except K12, and it had the highest values in F4K6, F2K8, and K10, which were not significantly different $(\mathrm{p}<0.05)$. However, the Kelvin-Voigt unit, $G_{2}$, reflected the resistance to deformation due to the three-dimensional network structure, and the highest $G_{2}$ values occurred in F10 and F8K2. Overall, F4K6, F2K8, and K10 exhibited similar gelling properties, the highest instantaneous moduli, and high retarded moduli.
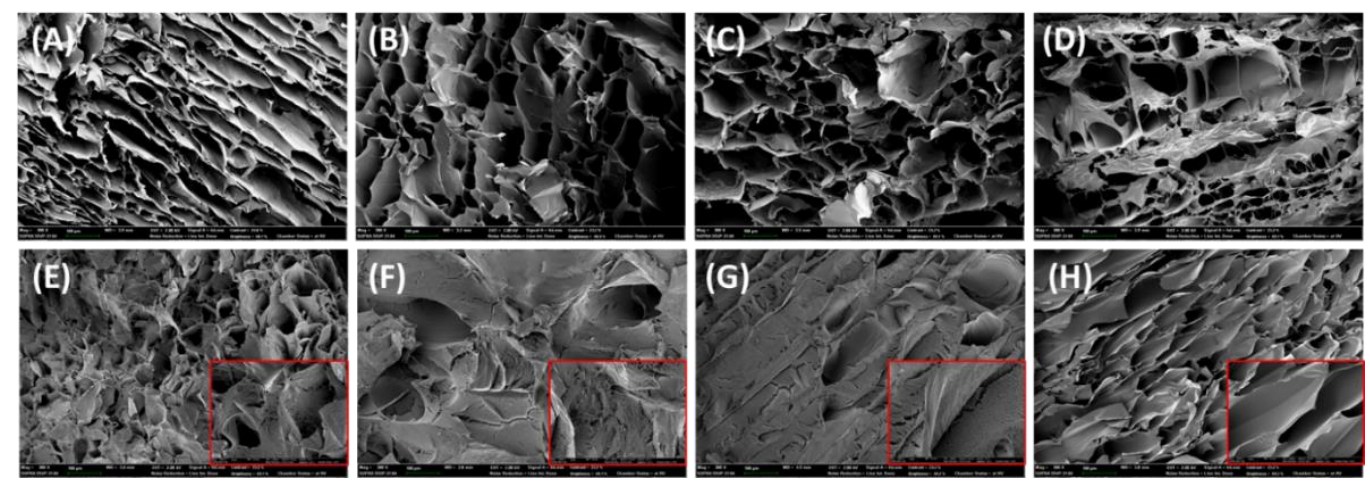

Figure 4. Microstructure of the compound gels, including (A) F12; (B) F10; (C) F8K2; (D) F6K4; (E) F4K6; (F) F2K8; (G) K10; and (H) K12 via SEM images. Samples were shown at magnification of $300 \times$ and $2,000 \times$, which were in the red box.

\subsection{Scanning electron microscopy analysis}

In order to analyze the rheological properties, the microstructures of the compound gels were confirmed by SEM. Figure 4 shows the microstructure of the compound gels that contained different ratios among FSG, KGM, and AG. In the case of the test groups with only FSG and KGM (Figures $3 \mathrm{~A}$ and B), the pores had a flat and smooth surface, and this might have been responsible for the low hardness values of the gels [27]. However, in the gels in which FSG and KGM were mixed (Figures 3B to G), the pore was somewhat denser, and the surface became rougher as the KGM increased. The KGM molecules could interact with the FSG molecules by hydrogen bonding, which is expected to enhance the interaction between molecules and the formation of networks [36]. Results similar to these have been reported in the study of cyclodextrin's effect on the properties of $\kappa$-carrageenan/KGM compound gels and in the study of the interaction between FSG and meat protein mixtures [27,37]. In addition, as shown in the red boxes of Figures $3 \mathrm{E}$ to $\mathrm{G}$, which were confirmed by increasing the resolution to 2,000 $\mathrm{x}$, networks of dense and small gel structures connected on the surfaces of the pores were observed in the F6K4, F8K2, and K10 samples. These microstructures could be due to the 
interaction between KGM and AG since it generally is known that the small and uniformly arranged pores could form a rigid structure [38,39]. This result also was expected to be related to a correlation between the ratio of KGM and the elasticity. In addition, the microstructures of F4K8 and F2K8, which exhibited molecular chain entanglements and dense structures, were thought to be associated with the capacity for excellent water retention. Since the gel sample had dense pores, the thin-walled structure was considered to have a large surface area and a much higher capillary force binding to water [40]. Therefore, these results were judged to be explanations of the result of F4K6 having a low minimum gelling concentration (Figure 1B), higher water holding capacity, and high interaction with water molecules (Table 1).

\subsection{FTIR characterization of compound gels}

A Fourier transform infrared spectroscopy study was conducted to analyze the rheological results to provide a clearer insight into the chemical interactions between the compound gel's biopolymers. Therefore, the FT-IR spectra of different ratios of FSG, $\mathrm{KGM}$, and AG were investigated in the $4,000-500 \mathrm{~cm}^{-1}$ region (Figure 5). The compound gels could form a three-dimensional, cross-linked, and continuous gel matrix by intermolecular and intra-molecular interactions, leading to a change in the viscoelastic property $[41,42]$. The spectral distributions of all of the samples of gel were nearly identical, indicating that the chemical structure of the compound gels with different proportions essentially had remained unchanged. Nevertheless, the most marked change occurred in the wavelength range of $1,640-1,590 \mathrm{~cm}^{-1}$, which could be allocated to the stretching of the associated hydroxyl group by $\mathrm{CO}$ [43]. As the ratio of KGM increased, the peak intensity increased to $1,640 \mathrm{~cm}^{-1}$, and, conversely, as the ratio of FSG increased, the peak intensity showed an increase to $1,590 \mathrm{~cm}^{-1}$. These results were similar to the shift of the peak due to the strong intermolecular hydrogen bonding interaction between FSG and KGM, which was reported in a study by Y. Jiang et al. [24]. The broad and strong peaks in the area of $3,330-3,270 \mathrm{~cm}^{-1}$ with a peak at $3304 \mathrm{~cm}^{-1}$ was thought to be associated with the presence of the stretching vibrations of the free, inter-and intra-molecular hydroxyl groups that formed hydrogen bonds between the biopolymers $[43,44]$. The existence of asymmetrical weak stretching vibrations was observed in the range of $2,940-2,880 \mathrm{~cm}^{-1}$, which was thought to be related to the presence of a saturated bond of $\mathrm{C}-\mathrm{H}$ [45]. The sharp and strong peaks in the area of $1,032-1,017 \mathrm{~cm}^{-1}$ with a peak at $1029 \mathrm{~cm}^{-1}$ was attributed to the vibration stretching associated with the $\mathrm{CH}_{2}$ groups. 


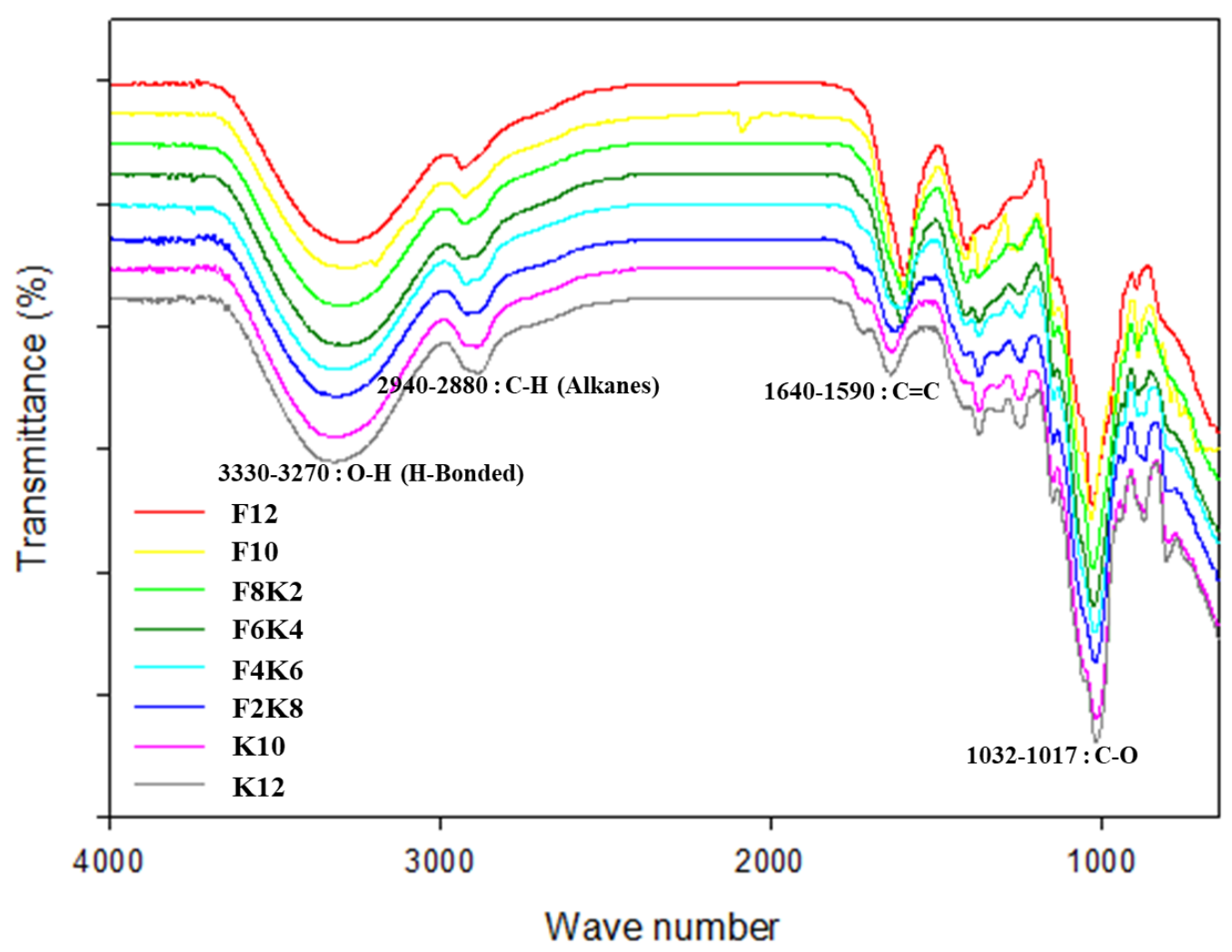

Figure 5. FTIR spectra of the compound gels, including different ratios of FSG, KGM, and AG.

\subsection{Structure-rheological property relationship for the compound gel}

The three components used in this study had different gelling properties. FSG had "weak gel-like" properties, depending on the macroscopic behavior. In contrast, KGM and AG, which can be characterized as "true gels," differ from each other in their gelation mechanisms. KGM is gelled by cooling (cold-set gelation), and AG is gelled by heating (heat-set gelation) [46]. In other words, AG forms a gelation structure by hydrogen bonds at low temperature, but it is known that the hydrogen bonds break when the temperature is increased, and gelation is released. On the contrary, it is known that gels that contain KGM form sol when the temperature is low and the gel forms as the temperature increases. This is related to the solvent's structural stability, and the entropy of the solvent increases as the temperature increases; as a result, it is known that gelation occurs as a structure is formed by hydrophobic interactions between the gel molecules. KGM has 5$10 \%$ of random acetyl groups in C- 6 , and it is known that gelation ability is increased by deacetylation [47]. In addition, as the temperature rises, gelation by hydrophobic interaction is possible. The proposed gel was homogenized while heating the three components in powder form to form a gel structure. The increase in the entropy as the temperature increases breaks all of the hydrogen bonds as well as the partial hydrophobic bonds of KGM that had formed partial gelation. After sufficient homogenization, if the temperature is decreased, it is thought that a collective structure will be formed by hydrogen bonding between AG, KGM, and FSG. Therefore, it was judged that more small structures were formed in the junction zone, which was the primary chain linkages that form a hierarchical structure in the gelation process of compound gels by hydrophobic interactions (red box in Figure 4). In addition, during the heat treatment process, the junction zone was formed by aggregation of the glucomannan chain in KGM due to hydrophobic interaction. During the process of decreasing the temperature, the junction zone can be formed more strongly 
by the hydrogen bonding of the biopolymers [3]. In particular, as the ratio of KGM increased, it was thought that more small structures were formed in the junction zone by hydrophobic interactions, resulting in the formation of a rigid gel and a relatively high water retention ability (Figure 6). These results also were confirmed indirectly by Yuan et al. [27]. These researchers confirmed that the higher the partial network formation of KGM by deacetylation is, the higher the surimi gel's elasticity will be. In addition, Zhang et al. (2015) showed that hydrophobic interactions with proteins can be improved by using the deacetylation of KGM by heat treatment, resulting in the generation of a dense and uniform microstructure of surimi gel [48].

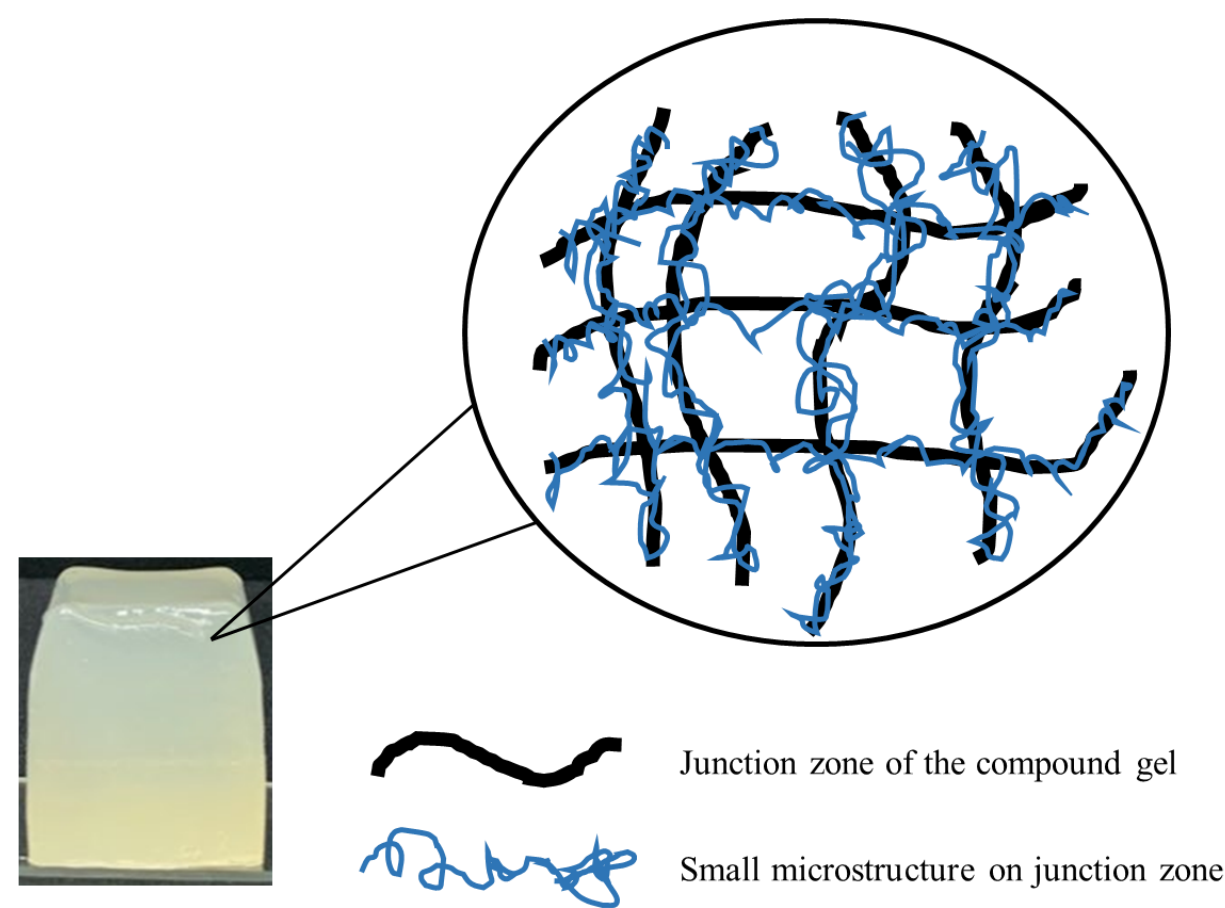

The elastic compound gel including FSG, KGM, and AG

Figure 6. Scheme of predictive microstructure of the compound gel including FSG, KGM, and AG.

\section{Conclusions}

In this study, compound gels were developed using flaxseed gum, konjac glucomannan, and agar for a high-moisture elastic gelling agent. The compound gels were prepared by mixing different quantities of FSG and KGM with a fixed ratio of AG. We investigated the physicochemical properties and microstructures of compound gels, including MGC, WBC, WSI, SP, texture analysis, and rheological analysis. The samples of F6K4 and F4K6 were significantly different $(p<0.05)$ in WBC and SP, indicating that these gels could entrap water effectively when exposed to external force, and the interaction between the gel and water was the strongest. The results of the texture and rheological analyses indicated that F4K6, F2K8, and $\mathrm{K} 10$ had significantly high elastic values $(p<0.05)$. As a result of the analysis of the microstructure through SEM, it was determined that the high elastic properties of these gels were correlated with their having a small and dense structure in the junction zone. Overall, F4K6 had both good water retention and good elasticity. This might be because FSG had both good water holding and binding capacities, and KGM 
could improve the hydrogen bonds of the junction zones through heat treatment. Also, the addition of a small amount of AG could provide the viscoelastic gel with physical entanglement and a solid structure. These results suggested that the F4K6 could be used in synergistic interactions, which promote elastic properties, water binding capacity, and a compact porous microstructure.

Supplementary Materials: The following are available online at www.mdpi.com/xxx/s1, Figure S1: Mechanical model used to describe the rheological properties of proposed gels: (a) Burger's model; (b) a creep and recovery plot described by Burger's model.

Author Contributions: Conceptualization, J. Hahn and Y. J. Choi.; methodology, J. Hahn; software, Y. Yang; validation, J. Yang, J. Hahn and Y. J. Choi.; formal analysis, J. Hahn, and J. Kim; investigation, J. Kim.; resources, J. Kim.; data curation, J. Yang.; writing, i.e., preparation of the original draft, J. Hahn and J. Yang; writing - review and editing, J. Hahn and Y. J. Choi; visualization, J. Hahn; supervision, Y. Choi; project administration, J. Kim; funding acquisition, Y. J. Choi and J. Kim. All of the authors have read and agreed to the published version of the manuscript.

Funding: This research was supported by the Technology Development Program (S2777815), funded by the Ministry of SMEs and Startups (MSS, Korea).

Conflicts of Interest: The authors declare no conflict of interest. 


\section{References}

1. Dickinson, E. Hydrocolloids at interfaces and the influence on the properties of dispersed systems. Food hydrocolloids 2003, $17,25-39$.

2. Banerjee, S.; Bhattacharya, S. Food gels: gelling process and new applications. Critical reviews in food science and nutrition 2012, 52, 334-346.

3. Saha, D.; Bhattacharya, S. Hydrocolloids as thickening and gelling agents in food: a critical review. Journal of food science and technology 2010, 47, 587-597.

4. Qian, K.; Cui, S.; Wu, Y.; Goff, H. Flaxseed gum from flaxseed hulls: Extraction, fractionation, and characterization. Food Hydrocolloids 2012, 28, 275-283.

5. Ziolkovska, A. Laws of flaxseed mucilage extraction. Food Hydrocolloids 2012, 26, 197-204.

6. Zhao, Q.; Long, Z.; Kong, J.; Liu, T.; Sun-Waterhouse, D.; Zhao, M. Sodium caseinate/flaxseed gum interactions at oil-water interface: Effect on protein adsorption and functions in oil-in-water emulsion. Food Hydrocolloids 2015, 43, 137-145.

7. Cui, W.; Mazza, G.; Biliaderis, C. Chemical structure, molecular size distributions, and rheological properties of flaxseed gum. Journal of Agricultural and Food Chemistry 1994, 42, 1891-1895.

8. Wu, M.; Li, D.; Wang, L.-j.; Zhou, Y.-g.; Mao, Z.-h. Rheological property of extruded and enzyme treated flaxseed mucilage. Carbohydrate Polymers 2010, 80, 460-466.

9. Chen, H.-H.; Xu, S.-Y.; Wang, Z. Gelation properties of flaxseed gum. Journal of food engineering 2006, 77, 295-303.

10. Fodje, A.M.; Chang, P.R.; Leterme, P. In vitro bile acid binding and short-chain fatty acid profile of flax fiber and ethanol co-products. Journal of medicinal food 2009, 12, 1065-1073.

11. Mazza, G.; Biliaderis, C. Functional properties of flax seed mucilage. Journal of Food Science 1989, 54, 1302-1305.

12. Hu, Y.; Tian, J.; Zou, J.; Yuan, X.; Li, J.; Liang, H.; Zhan, F.; Li, B. Partial removal of acetyl groups in konjac glucomannan significantly improved the rheological properties and texture of konjac glucomannan and $\kappa$-carrageenan blends. International journal of biological macromolecules 2019, 123, 1165-1171.

13. Devaraj, R.D.; Reddy, C.K.; Xu, B. Health-promoting effects of konjac glucomannan and its practical applications: A critical review. International journal of biological macromolecules 2019, 126, 273-281.

14. Lafarge, C.; Cayot, N.; Hory, C.; Goncalves, L.; Chassemont, C.; Le Bail, P. Effect of konjac glucomannan addition on aroma release in gels containing potato starch. Food research international 2014, 64, 412-419.

15. Zhang, Y.-q.; Xie, B.-j.; Gan, X. Advance in the applications of konjac glucomannan and its derivatives. Carbohydrate Polymers 2005, 60, 27-31.

16. Armisen, R.; Galatas, F. Production, properties and uses of agar. Production and utilization of products from commercial seaweeds. FAO Fish. Tech. Pap 1987, 288, 1-57.

17. Williams, P.W.; Phillips, G.O. Chapter 2: Agar. Handbook of hydrocolloids. Cambridge: Woodhead 2000, $501,28$.

18. Cui, W.; Mazza, G.; Oomah, B.; Biliaderis, C. Optimization of an aqueous extraction process for flaxseed gum by response surface methodology. LWT-Food Science and Technology 1994, 27, 363-369.

19. Wang, X.; Reddy, C.K.; Xu, B. A systematic comparative study on morphological, crystallinity, pasting, thermal and functional characteristics of starches resources utilized in China. Food chemistry 2018, 259, 81-88.

20. Zhang, H.; Zhang, F.; Yuan, R. Applications of natural polymer-based hydrogels in the food industry. In Hydrogels Based on Natural Polymers; Elsevier: 2020; pp. 357-410.

21. Reháková, M.; Bakoš, D.; Soldán, M.; Vizárová, K. Depolymerization reactions of hyaluronic acid in solution. International journal of biological macromolecules 1994, 16, 121-124.

22. Olais-Govea, J.M.; López-Flores, L.; Chávez-Páez, M.; Medina-Noyola, M. Nonequilibrium kinetics of the transformation of liquids into physical gels. Physical Review E 2018, 98, 040601.

23. Connolly, M.L.; Lovegrove, J.A.; Tuohy, K.M. Konjac glucomannan hydrolysate beneficially modulates bacterial composition and activity within the faecal microbiota. Journal of Functional Foods 2010, 2, 219-224. 
24. Jiang, Y.; Reddy, C.K.; Huang, K.; Chen, L.; Xu, B. Hydrocolloidal properties of flaxseed gum/konjac glucomannan compound gel. International journal of biological macromolecules 2019, 133, 1156-1163.

25. Sangnark, A.; Noomhorm, A. Effect of particle sizes on functional properties of dietary fibre prepared from sugarcane bagasse. Food chemistry 2003, 80, 221-229.

26. Yuan, Y.; Wang, L.; Mu, R.-J.; Gong, J.; Wang, Y.; Li, Y.; Ma, J.; Pang, J.; Wu, C. Effects of konjac glucomannan on the structure, properties, and drug release characteristics of agarose hydrogels. Carbohydrate polymers 2018, 190, 196-203.

27. Yuan, C.; Xu, D.; Cui, B.; Wang, Y. Gelation of $\kappa$-carrageenan/Konjac glucommanan compound gel: Effect of cyclodextrins. Food Hydrocolloids 2019, 87, 158-164.

28. Thrimawithana, T.; Young, S.; Dunstan, D.; Alany, R. Texture and rheological characterization of kappa and iota carrageenan in the presence of counter ions. Carbohydrate Polymers 2010, 82, 69-77.

29. Maurer, S.; Junghans, A.; Vilgis, T.A. Impact of xanthan gum, sucrose and fructose on the viscoelastic properties of agarose hydrogels. Food Hydrocolloids 2012, 29, 298-307.

30. Lefeuvre, A.; Le Duigou, A.; Bourmaud, A.; Kervoelen, A.; Morvan, C.; Baley, C. Analysis of the role of the main constitutive polysaccharides in the flax fibre mechanical behaviour. Industrial Crops and Products 2015, 76, 1039-1048.

31. Kurt, Ş.; Ceylan, H.G. Effects of flaxseed and pH on the emulsion properties of beef by using a model system. Turkish Journal of Agriculture-Food Science and Technology 2018, 6, 78-83.

32. Nordqvist, D.; Vilgis, T.A. Rheological study of the gelation process of agarose-based solutions. Food Biophysics 2011, 6, 450460 .

33. Qiao, L.; Li, Y.; Chi, Y.; Ji, Y.; Gao, Y.; Hwang, H.; Aker, W.G.; Wang, P. Rheological properties, gelling behavior and texture characteristics of polysaccharide from Enteromorpha prolifera. Carbohydrate polymers 2016, 136, 1307-1314.

34. Skinner, G.; Rao, V. Linear viscoelastic behavior of frankfurters. Journal of texture studies 1986, 17, 421-432.

35. Sozer, N. Rheological properties of rice pasta dough supplemented with proteins and gums. Food Hydrocolloids 2009, 23, 849855.

36. Gong, J.; Wang, L.; Wu, J.; Yuan, Y.; Mu, R.-J.; Du, Y.; Wu, C.; Pang, J. The rheological and physicochemical properties of a novel thermosensitive hydrogel based on konjac glucomannan/gum tragacanth. Lwt 2019, 100, 271-277.

37. Chen, H.-H.; Xu, S.-Y.; Wang, Z. Interaction between flaxseed gum and meat protein. Journal of Food Engineering 2007, 80, 1051-1059.

38. Hermansson, E.; Schuster, E.; Lindgren, L.; Altskär, A.; Ström, A. Impact of solvent quality on the network strength and structure of alginate gels. Carbohydrate polymers 2016, 144, 289-296.

39. Liu, S.; Li, L. Thermoreversible gelation and scaling behavior of Ca2+-induced $\kappa$-carrageenan hydrogels. Food Hydrocolloids 2016, 61, 793-800.

40. Mao, R.; Tang, J.; Swanson, B. Water holding capacity and microstructure of gellan gels. Carbohydrate polymers 2001, 46, 365371.

41. Amarioarei, G.; Spiridon, I.; Lungu, M.; Bercea, M. Rheological investigation of Prunus Sp. gums in aqueous medium. Industrial \& engineering chemistry research 2011, 50, 14148-14154.

42. Rubinstein, M.; Dobrynin, A.V. Associations leading to formation of reversible networks and gels. Current opinion in colloid $\mathcal{E}$ interface science 1999, 4, 83-87.

43. Li, B.; Li, J.; Xia, J.; Kennedy, J.; Yie, X.; Liu, T. Effect of gamma irradiation on the condensed state structure and mechanical properties of konjac glucomannan/chitosan blend films. Carbohydrate Polymers 2011, 83, 44-51.

44. Skwarek, E.; Goncharuk, O.; Sternik, D.; Janusz, W.; Gdula, K.; Gun'ko, V.M. Synthesis, structural, and adsorption properties and thermal stability of nanohydroxyapatite/polysaccharide composites. Nanoscale research letters 2017, 12, 1-12.

45. Alzorqi, I.; Sudheer, S.; Lu, T.-J.; Manickam, S. Ultrasonically extracted $\beta$-d-glucan from artificially cultivated mushroom, characteristic properties and antioxidant activity. Ultrasonics sonochemistry 2017, 35, 531-540. 
46. Lafarge, C.; Cayot, N. Potential use of mixed gels from konjac glucomannan and native starch for encapsulation and delivery of aroma compounds: A review. Starch-Stärke 2018, 70, 1700159.

47. Du, X.; Li, J.; Chen, J.; Li, B. Effect of degree of deacetylation on physicochemical and gelation properties of konjac glucomannan. Food Research International 2012, 46, 270-278.

48. Zhang, T.; Xue, Y.; Li, Z.; Wang, Y.; Xue, C. Effects of deacetylation of konjac glucomannan on Alaska Pollock surimi gels subjected to high-temperature (120 C) treatment. Food Hydrocolloids 2015, 43, 125-131. 